\title{
Experiencia en el manejo de la patología del conducto tirogloso: serie de casos
}

\author{
Experience in thyroglossal duct pathology: clinical case series
}

\author{
Dr. Patricio Cieri ${ }^{a}$ Dra. Julia E. Udaquiola ${ }^{a}$, Dr. Santiago E. Calello y Dr. Daniel H. Liberto ${ }^{a}$
}

\begin{abstract}
RESUMEN
La patología del conducto tirogloso constituye la segunda anomalía cervical benigna más frecuente en la infancia. El diagnóstico es principalmente clínico. Sistrunk fue quien describió la técnica quirúrgica considerada de elección para el tratamiento definitivo de esta patología.

Estudio descriptivo retrospectivo. Entre junio de 2008 y agosto de 2015 se realizaron 54 procedimientos en 45 pacientes (39 casos primarios). La mediana de edad fue de 4,7 años; $14 / 39$ pacientes $(31,1 \%)$ presentaron infección preoperatoria. Todoslos pacientes fueron evaluados inicialmente por ecografía. Todos fueron tratados mediante la técnica de Sistrunk. La recidiva global fue de $17,8 \%$.

Palabras clave: quiste tirogloso, quiste cervical, técnica Sistrunk.
\end{abstract}

\begin{abstract}
The thyroglossal duct cyst pathology represents the second cause of bening cervical anomalies in childhood. Diagnosis is mainly clinical. Sistrunk (1920) proposed a surgical technique that is still considered the gold standard for definitive treatment of this condition.

A retrospective study was made including patients who underwent surgery for thyroglossal duct cyst pathology in our department between June 2008 and August 2015. In this period, we performed 54 procedures in 45 patients ( 39 primary cases). Median age was 4.7 years; $14 / 39$ patients (31.1\%) had pre-operative infection. All patients were studied with neck ultrasound. A Sistrunk`s procedure was performed in all cases. The global recurrence rate was $17.8 \%(8 / 45)$.

Key words: thyroglossal cyst, cervical cyst, Sistrunk technique.
\end{abstract}

http:/ /dx.doi.org/10.5546/aap.2016.e310

a. Servicio de Cirugía Pediátrica. Hospital Italiano de Buenos Aires.

Correspondencia:

Dr. Patricio Cieri: patricio.cieri@hospitalitaliano.org.ar

Financiamiento: Ninguno.

Conflicto de intereses: Ninguno que declarar.

Recibido: 22-9-2015

Aceptado: 9-3-2016

\section{INTRODUCCIÓN}

La patología del conducto tirogloso (PCT) constituye la segunda anomalía cervical benigna más frecuente en pediatría, luego de las adenopatías, que representan el $70 \%-75 \%{ }^{1,2}$ El esbozo de la glándula tiroides aparece en la $4^{\text {ta }}$ semana de gestación. ${ }^{3}$ Durante su descenso hasta la porción baja del cuello, el esbozo medial se elonga y forma el conducto tirogloso (CT). Su involución se completa entre la $7^{\mathrm{ma}}$ y la $10^{\mathrm{ma}}$ semana. ${ }^{1}$ La falta de obliteración del CT antes de la formación mesodérmica del hueso hioides deviene en su persistencia. ${ }^{2}$ La PCT puede encontrarse en cualquier punto del trayecto de migración del primordio desde el foramen ciego en la lengua hasta el istmo de la glándula tiroides. ${ }^{4}$ Cuando se localiza sobre el cartílago tiroideo, generalmente, se encuentra lateralizado, más frecuente a la izquierda.

Según la relación con el hioides, el quiste puede encontrarse suprahioideo $(23 \%)$, en la región hioidea $(60 \%)$ (anterior o posterior a él) o infrahioideo (13\%). Los quistes intralinguales representan el 3\%. ${ }^{2}$ Las variantes anatómicas, en la literatura, se mencionan como reporte de casos, y no se puede establecer su verdadera incidencia. Se destacan, además del quiste intralingual, la comunicación con el lóbulo piramidal de la tiroides y la fusión tirohioidea. En ocasiones, puede infectarse y es necesaria antibioticoterapia y/o drenaje previo al tratamiento definitivo.

\section{POBLACIÓN Y MÉTODOS}

Estudio descriptivo retrospectivo. Se incluyeron pacientes de entre 0 y 18 años de edad, con diagnóstico histopatológico de PCT, operados en el Servicio de Cirugía Pediátrica del Hospital Italiano de Buenos Aires entre junio de 2008 y agosto de 2015. Se revisaron las bases de datos y las historias clínicas electrónicas. Se analizaron las siguientes variables: sexo, edad, infección preoperatoria, porcentaje de recidiva, estudios complementarios, técnica quirúrgica, estadía posoperatoria y variantes anatómicas. Nuestro protocolo diagnóstico se basa en el examen físico y la ecografía en todos los pacientes. 
Se realizó, en todos los casos, la técnica de Sistrunk. Se analizaron los resultados luego de un período de seguimiento promedio de 7 meses (r: 1-53 m).

En pacientes con infección preoperatoria, se indicó el tratamiento antibiótico (cefalosporina de $1^{a}$ generación) por 15 días y se programó al mes la cirugía, con profilaxis 7 días antes de la intervención. Se definieron como casos primarios a aquellos que fueron operados por primera vez en nuestro Servicio y secundarios a aquellos con cirugía inicial realizada en otro centro. La cirugía se realiza de forma ambulatoria y se deja un drenaje por 24 horas, excepto las recidivas múltiples. Se obtuvo aprobación del Comité de Ética del Hospital.

\section{CASOS CLÍNICOS}

Se analizó un período de 7 años. Se incluyeron 54 procedimientos en 45 pacientes. La mediana de edad fue de 4,7 años (r: 1,8-17), con una relación varón mujer 1:1. El 86,6\% de los casos $(n=39)$ fueron primarios.

De los 39 casos primarios, 25 (64,1\%) consultaron por una masa indolora, móvil, no inflamatoria, y $14(31,1 \%)$ presentaron antecedentes de infección (tumoración eritematosa dolorosa con aumento de la temperatura). El $83,3 \%(5 / 6)$ de los casos secundarios tuvieron infección preoperatoria (Tabla 1).

Estudios complementarios: ecografía compatible con PCT y glándula tiroides normotópica en todos los pacientes. Centellograma tiroideo $(n=4)$ y estudio de laboratorio con función tiroidea $(n=3)$, los cuales fueron pedidos antes de la derivación a nuestro Centro. Un paciente se estudió con tomografía computada por sospecha de patología esofágica. Tres pacientes se estudiaron con resonancia magnética (RMI) (una PCT intralingual y dos con múltiples recidivas).

TABLA 1. Generalidades y recidiva

\begin{tabular}{lcc}
\hline & \multicolumn{2}{c}{$\mathbf{N = 4 5}$} \\
\cline { 2 - 3 } & Casos 1, n= 39 & Casos 2, n= 6 \\
\hline Edad en años (media y rango) & $4,6(1,8-17)$ & $4,9(6-17)$ \\
Sexo (relación mujer/varón) & $1: 1$ & $1: 2$ \\
Variantes anatómicas & $3(7,6 \%)$ & 0 \\
Infección preoperatoria (n y \%) & $14(31,1 \%)$ & $5(83,3 \%)$ \\
Recidiva (n y \%) & $7(17,9 \%)$ & $1(16,6 \%)$ \\
Recidiva con infección previa & $2(14,2 \%)$ & $3(50 \%)$ \\
Recidiva global & \multicolumn{2}{c}{$17,8 \%(8 / 45)$} \\
\hline
\end{tabular}

El índice de recidiva (reaparición de la lesión en relación con el sitio quirúrgico) global fue de 17,8\% (8/45). Dos pacientes fueron recibidos luego de múltiples cirugías (r: 4-8). Si bien todos los pacientes se estudiaron con ecografía, ante recidivas múltiples, se realizó una resonancia. De los 14 pacientes con infección previa, solo 2 recidivaron, mientras que, de los 25 sin infección, 5 lo hicieron (Tabla 1). En los casos primarios recidivados, se realizó una reoperación (resección amplia de la lesión recidivada) por paciente. Entre los secundarios, el número de procedimientos realizados por nuestro equipo varió de 1 a 3 para lograr la resolución completa.

Si bien, habitualmente, los pacientes son manejados de forma ambulatoria, 15 procedimientos $(27,7 \%)$ requirieron 24 horas de internación: 8 fueron recidivas en casos secundarios, un caso de PCT intralingual, una variante anatómica (fusión tirohioidea) (Figura 1) que prolongó el tiempo operatorio y 5 por razones administrativas.

Reportamos 4 complicaciones menores ( 2 infecciones de heridas, un absceso y una cicatrización hipertrófica) y 9 mayores (una perforación de la membrana y 8 recidivas).

\section{DISCUSIÓN}

La edad de presentación de la PCT presenta una curva bimodal, con un pico a los 5-6 años

FIGURA 1. Imagen intraquirúrgica, que muestra ausencia de limite entre el hueso hioides (flecha blanca) y la membrana tirohioidea (fusión tirohioidea) por detrás (flecha punteada).

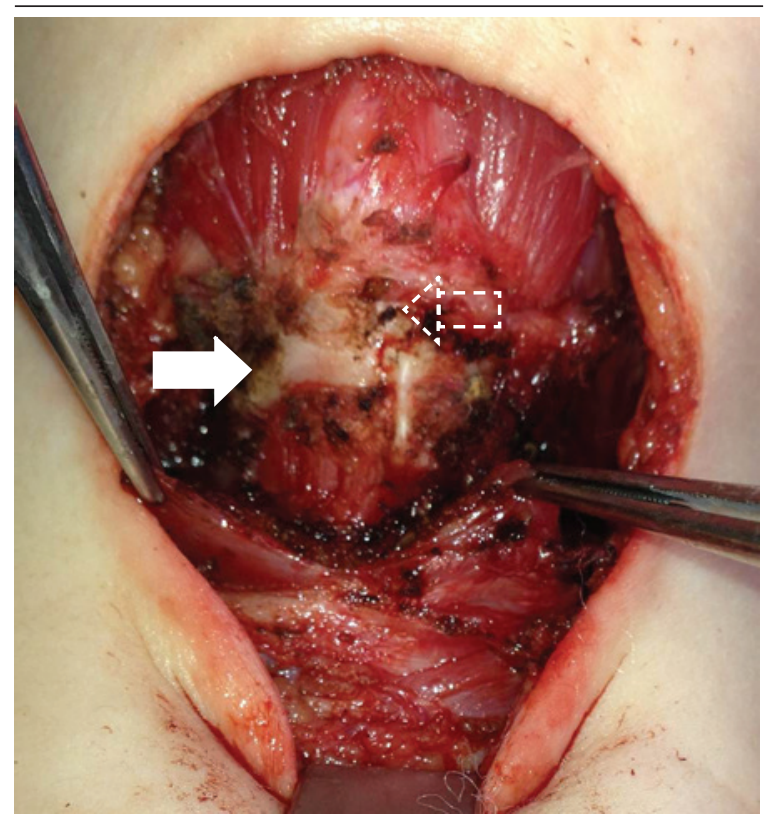


y el segundo en la adultez. ${ }^{2,4}$ La mediana de presentación de edad en nuestra serie fue de 4,7 años (r: 1,8-17). El seguimiento promedio fue 7 meses. No consideramos que esta sea una limitante, ya que la mayor parte de los autores concuerda que la amplia mayoría de las recidivas suceden dentro de las primeras semanas posoperatorias.

Habitualmente, la PCT se presenta como una tumoración en el área perihioidea $(98 \%),{ }^{5}$ renitente, adherida a planos superficiales, que asciende con la deglución, y el diagnóstico es fundamentalmente clínico. Otra forma de presentación es un orificio en la línea media, a través del cual puede salir material mucoso. Esta secreción puede volverse purulenta en casos de infección. ${ }^{6}$ La PCT intralingual se presenta con dificultad o molestia durante la deglución. En nuestro caso, el paciente consultó por disfagia progresiva con sospecha de faringitis.

Un tercio de los pacientes se manifiestan con infección, debida frecuentemente a Haemophilus influenzae, Staphylococcus epidermidis y aureus. ${ }^{2}$ En nuestra serie, el porcentaje de infecciones

FIgURA 2. Resonancia magnética: A. corte axial conadquisición T2, que muestra una imagen espontáneamente hiperintensa (quística) en la base de la lengua, que protruye hacia la faringe (flecha). B. corte axial con adquisición T1, control posquirúrgico que evidencia una cicatriz en la zona del quiste (flecha). C. corte sagital, adquisición T1, donde se observa la lesión quística mencionada previamente en la base de la lengua (flecha). D. corte sagital en adquisición T2, que pone en evidencia el tejido cicatrizal posquirúrgico en la región mencionada.

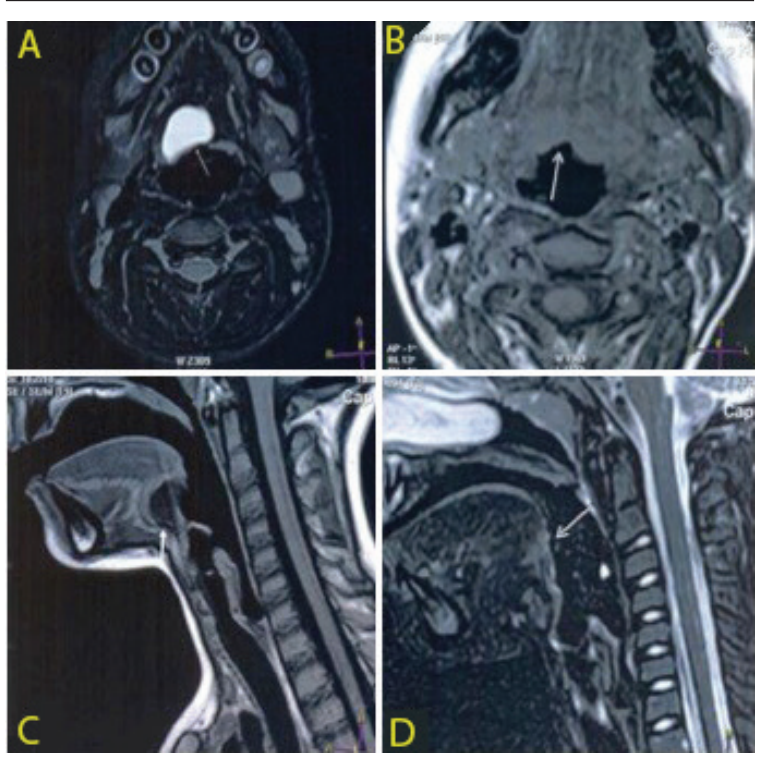

ascendió a 42,2\%. Series similares ${ }^{7-9}$ reportan una frecuencia de $22 \%, 55 \%$ y $61 \%$, respectivamente. Kaselas et al. ${ }^{9}$ inician el tratamiento médico y posponen 3 meses la cirugía. En ambos casos, la antibioticoterapia se continúa hasta 7 días después de la operación.

El método diagnóstico más utilizado es la ecografía, que permite caracterizar, en la mayoría de los casos, la presencia de la glándula tiroides en su localización normal ${ }^{1}$ (Figura 2). El estudio de laboratorio de función tiroidea y el centellograma ya no se realizan de rutina, dado que no aportan datos significativos en pacientes con ecografía tiroidea normal. La RMI brinda mayor información anatómica y del compromiso de partes blandas; se realiza de rutina ante la sospecha de PCT intralingual (Figura 3) y en pacientes con múltiples recidivas (Figura 4).

En 1920, Sistrunk describió la técnica de resección del tejido patológico hasta la base de la lengua. Tomando como modelo esta técnica, se reseca la porción central del hioides hasta las astas laterales y se amplía la resección de tejido sano. Se considera que esto disminuye los posibles remanentes de tejido patológico. ${ }^{10}$

FIGURA 3. Ecografía cervical (con modalidad doppler), que muestra una lesión quística inmediatamente por debajo de la piel. Nótese la relación con la glándula tiroides por debajo (flecha).

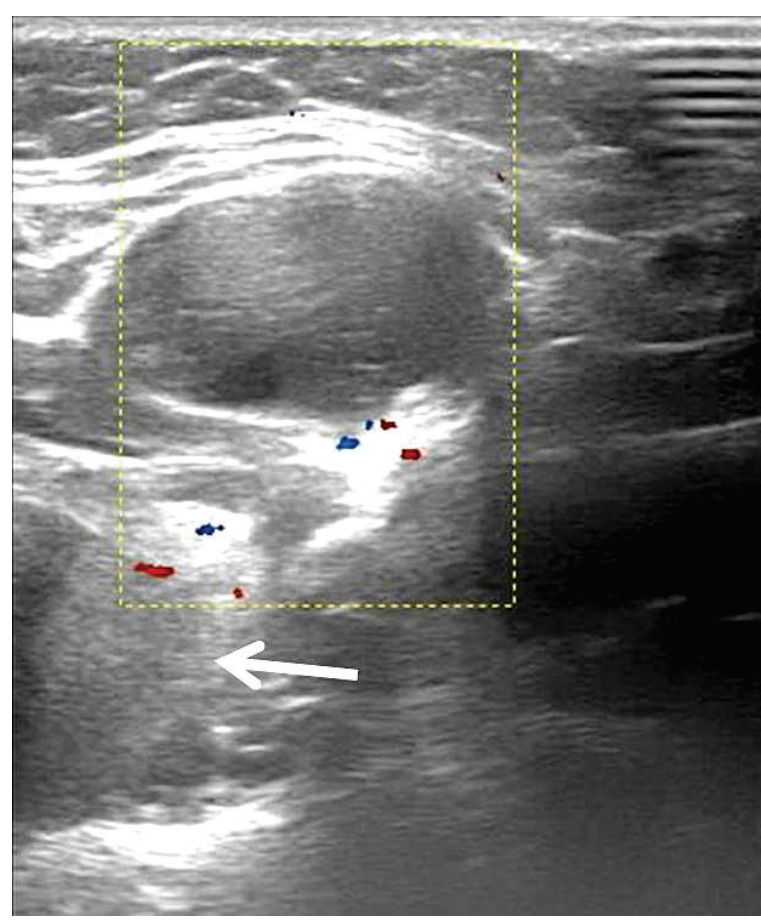


Un metaanálisis reciente ${ }^{11}$ reporta índices de recurrencia de $10,7 \%$ para los casos primarios y $20 \%$ para los secundarios. Hussain ${ }^{12}$ expone, en su serie, $85 \%$ de infección pre- o perioperatoria, con un índice de recidiva de 3,9\%. Kaselas ${ }^{9}$ muestra una marcada relación entre la infección preoperatoria y el índice de recidiva. Contrariamente, la tasa de recidiva en nuestra serie fue mayor en los pacientes sin antecedentes de infección. Esta podría ser una limitante debido al número de la muestra. Marianowski ${ }^{13}$ propone 4 factores de riesgo preponderantes: menores de dos años, más de dos episodios de infección, drenaje previo y múltiples quistes en la anatomía patológica. Estos factores no fueron considerados al momento de la recolección de datos. Sin embargo, deberían ser tenidos en cuenta para un futuro análisis.

Históricamente, la cirugía de cabeza y cuello era considerada poco segura para realizarse en forma ambulatoria. Bratu et al., ${ }^{14}$ reportan un $46 \%$ de cirugía ambulatoria. Estos porcentajes varían según haya operado un cirujano pediátrico o un otorrinolaringólogo y la utilización de drenaje posoperatorio.

En general, las complicaciones no son graves, aunque algunas revisten mayor complejidad. Maddalozzo ${ }^{15}$ las divide en mayores y menores; estas últimas están relacionadas, principalmente, con la infección de la herida. En dicha serie, el $29 \%$ son menores. No reporta complicaciones mayores (recidiva, perforación de la membrana tirohioidea, hematomas).

\section{CONCLUSIÓN}

Ante la aparición de un nódulo cervical en línea media, tanto el pediatra como el cirujano

FIgURA 4. Resonancia magnética, corte axial en secuencia T2, en un paciente con múltiples cirugías previas, que evidencia lo siguiente: A. lesión hiperintesa que corresponde al trayecto patológico izquierdo (flecha); $B$. lesión hipointensa que corresponde a la región cicatrizal residual en íntima relación con el lóbulo izquierdo de la glándula tiroides (flecha).

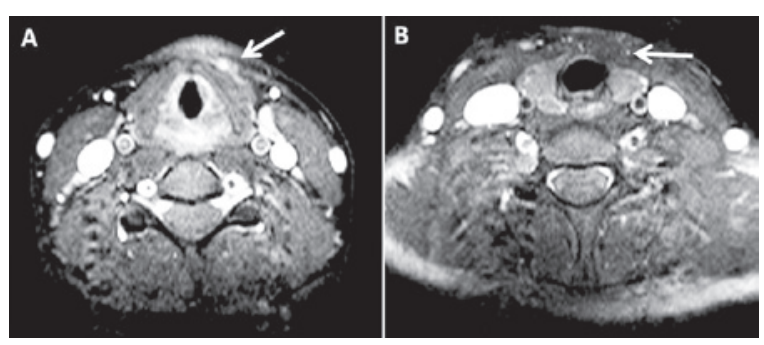

deben maximizar los esfuerzos para un correcto diagnóstico y tratamiento oportuno, antes de que se produzca la infección. Se recomienda sistematizar el diagnóstico y la técnica quirúrgica para la PTC, y realizar, en los casos infectados, un tratamiento antibiótico efectivo y el procedimiento diferido para disminuir así las complicaciones intra- y posoperatorias y el índice de recidiva. La operación de Sistrunk es la técnica de elección en procedimientos primarios; aún así, la recidiva continúa siendo elevada, y se debe notificar al paciente y a su familia previamente.

\section{Agradecimientos}

A la Dra. Anahí Salomón (crítica) y al Dr. Pablo Lobos (crítica).

\section{REFERENCIAS}

1. Chou J, Walters A, Hage R, Zuranda A, et al. Thyroglossal duct cysts: anatomy, embryology and treatment. Surg Radiol Anat 2013;35(10):875-81.

2. Azizkhan RG. Preface. Semin Pediatr Surg 2006;15(2):63. [Acceso: 18 de enero de 2014]. Disponible en: http:/ / www. sempedsurg.org/article/S1055-8586\%2806\%2900013-8/pdf.

3. Sadler TW. Cabeza y cuello. In Sadler TW (ed). Langman's Medical Embryology. 6th ed. Baltimore, MD: Williams and Wilkins; 1990.Págs.312-3.

4. Organ GM, Organ CH Jr. Thyroid Gland and Surgery of the Thyroglossal Duct : Exercise in Applied Embryology. World J Surg 2000; 24(8):886-90.

5. Maddalozzo J, Alderfer J, Modi V. Posterior Hyoid Space as Related to Excision of the Thyroglossal Duct Cyst. Laryngoscope 2010;120(9):1773-8.

6. Palomo Luna J, de la Fé Soca AM, Bestard Hartman IC, Ramirez Salinas YM, Suarez Lescay C. Fístula tiroglosa con trayecto infrecuente. Medisan (Santiago de Cuba) 2012;16(9):1451-6.

7. Ostlie DJ, Burjonrappa SC, Snyder CL, Watts J, et al. Thyroglossal duct infections and surgical outcomes. J Pediatr Surg 2004;39(3):396-9.

8. Athow AC, Fagg NL, Drake DP. Management of thyroglossal cysts in children. Br J Surg 1989;76(8):811-4.

9. Kaselas C, Tsikopoulos G,Chortis C, Kaselas B. Thyroglossal duct cyst's inflammation. When do we operate? Pediatr Surg int 2005;21(12):991-3.

10. Horisawa M, Ninomi N, Nishimoto K, Matsunaga K, et al. Clinical results of the shallow core-out procedure in thyroglossal duct cyst operation. J Pediatr Surg 1999;34(11):1589-92.

11. Galluzzi F, Pingnataro L, Gaini RM, Hartley B, Garavello W. Risk of recurrence in children operated for thyroglossal duct cysts: A systematic review. J Pediatr Surg 2013;48(1):222-7.

12. Hussain K, Henney S, Tzifa K. A ten-year experience of thyroglossal duct cyst surgery in children. Eur Arch Otorhinolaryngol 2013;270(11):2959-61.

13. Marianowski R, Ait Amer JL, Morisseau-Durand MP, Manach Y, Rassi S. Risk factors for thyroglossal duct remnants after Sistrunk procedure in a pediatric population. Int J Pediatr Otorhinolaryngol 2003;67(1):19-23.

14. Bratu I, Laberge JM. Day surgery for thyroglossal duct cyst excision: a safe alternative. Pediatr Surg Int 2004;20(9):675-8.

15. Maddalozzo J, Venkatesan TK, Grupta P. Complications associated with the Sistrunk procedure. Laryngoscope 2001;111(1):119-23. 